\title{
A low-power transmitter design for inductive data link with class-F switching operation
}

\author{
Shinya Itoh ${ }^{1 \mathrm{a})}$ and Shoji Kawahito ${ }^{2 \mathrm{~b})}$ \\ ${ }^{1}$ Graduate School of Electronic Science and Technology, Shizuoka University \\ ${ }^{2}$ Research Institute of Electronics, Shizuoka University \\ a)sito@idl.rie.shizuoka.ac.jp \\ b) kawahito@idl.rie.shizuoka.ac.jp
}

\begin{abstract}
This letter presents a new design method of a low-power transmitter for inductive link. The proposed method introduces the concept of class-F RF amplifiers into an inductive data link. The power dissipation of the class-F transmitter is analyzed for carrier and symbol transition. It is revealed that the proposed transmitter consumes extremely low power of less than $0.2 \mathrm{~nJ} /$ bit with BPSK modulation at $2.5 \mathrm{M}$ Sample/s and the communication distance of about $50 \mathrm{~cm}$.
\end{abstract}

Keywords: inductive data link, class-F amplifier, harmonic tuning, low power communication, wireless data transmitter

Classification: Electron devices

\section{References}

[1] M. Ghovanloo and K. Najafi, "A wideband frequency-shift keying wireless link for inductively powered biomedical implants," IEEE Trans. Circuits Syst., vol. 51, no. 12, pp. 2374-2383, Dec. 2004.

[2] Y. Hu and M. Sawan, "A fully integrated low-power BPSK demodulator for implantable medical devices," IEEE Trans. Circuits Syst., vol. 52, no. 12, pp. 2252-2562, Dec. 2005.

[3] S. Y. Lee and S. C. Lee, "An implantable wireless bidirectional communication microstimulator for neuromuscular stimulation," IEEE Trans. Circuits Syst., vol. 52, no. 12, pp. 2526-2538, Dec. 2005.

[4] C. Sauer, M. Stanacevic, G. Cauwenberghs, and N. Thakor, "Power harvesting and telemetry in CMOS for implanted devices," IEEE Trans. Circuits Syst., vol. 52, no. 12, pp. 2605-2613, Dec. 2005.

[5] G. Iddan, G. Meron, A. Glukhovsky, and P. Swain, "Wireless Capsule Endoscopy," Nature, vol. 405, p. 417, May 2000.

[6] S. Itoh, S. Kawahito, and S. Terakawa, "A CMOS one-chip wireless camera with digital image transmission function for capsule endoscopes," IEEJ Trans. Sensors and Micromachines, vol. 126, no. 7, pp. 318-324, July 2006.

[7] F. H. Raab, "Class-F power amplifiers with maximally flat waveforms," IEEE Trans. Microwave Theory Tech., vol. 45, no. 11, pp. 2007-2012, Nov. 1997. 


\section{Introduction}

Recent interest in human activity monitoring has spurred a great deal of research into the implantable sensor devices. The integration of an ultralow-power and miniaturized wireless transmitter, which can be integrated on a single silicon SoC (System-on-a-chip) using CMOS technology, has become a crucial factor in the design of implantable devices.

An inductive link between two magnetically coupled coil is well known for a low-power communication method in implantable systems and it is easy to implement. In recent years, various methods employing inductive links have been discussed $[1,2,3,4]$. The desired data transmission rate is dependent on its applications. For example, capsule endoscope systems [5] require the data transmission rate of several mega bit per second. RF transmitters for such a purpose are required to have not only low-power consumption, but also high data transmission rate. However, the data transmission rate of the conventional inductive data link systems is limited to the range of $25 \mathrm{kbps}$ to 250 kbps.

The authors have recently reported an efficient transmitter design for inductive data link as an uplink communication method for capsule endoscopes [6] and demonstrated that the result of the total power consumption is $1.4 \mathrm{~mW}$ at the data transmission rate of $2.5 \mathrm{Mbps}$ with BPSK modulation (20 MHz carrier) and achieved the error-free image transmission over a distance of $48 \mathrm{~cm}$. The energy consumption of $0.56 \mathrm{~nJ} /$ bit has been achieved. This letter proposes a new design method of further low-power transmitters which introduces the concept of class-F amplifiers into an inductive data link for the first time.

\section{Transmitter design for inductive data link with class-F switch- ing operation}

\subsection{Circuit configuration}

Fig. 1 (a) shows the circuit schematic of the proposed transmitter. The data communication is established by inductive coupling between the transmitter coil and a receiver coil. The primary coil driver is implemented with a class-F tuned power amplifier. The class-F amplifier uses an output filter to control the harmonic content of its drain-voltage or drain-current waveforms, thereby shaping them to reduce power dissipation by the transistor and thus to increase efficiency. It does not require any linear amplifiers that consume large power and silicon area, and a simple CMOS inverter can be used as a switching device. Parallel LC resonators of the first through $N$ th stages are tuned to the first through $N$ th odd harmonics of the carrier frequency, respectively. An additional resistor $\mathrm{R}$ and a capacitor $\mathrm{C}$ are connected in series for peak current regulation and input DC current suppression, respectively.

In conventional class-F RF transmitters [7], the power is dissipated by a radiation resistance of antenna. In the proposed class- $\mathrm{F}$ transmitter for inductive link, the supply current is used for driving transmitter coil, $L_{1}$. The output LC tank is tuned to resonance at the carrier frequency. At the 


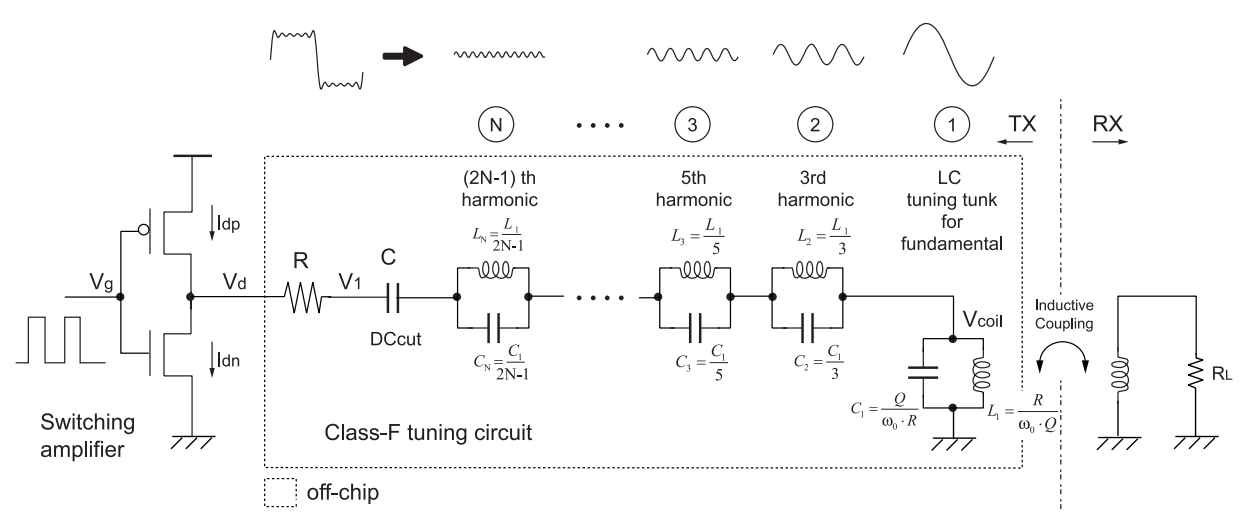

(a) Circuit schematic of the proposed transmitter
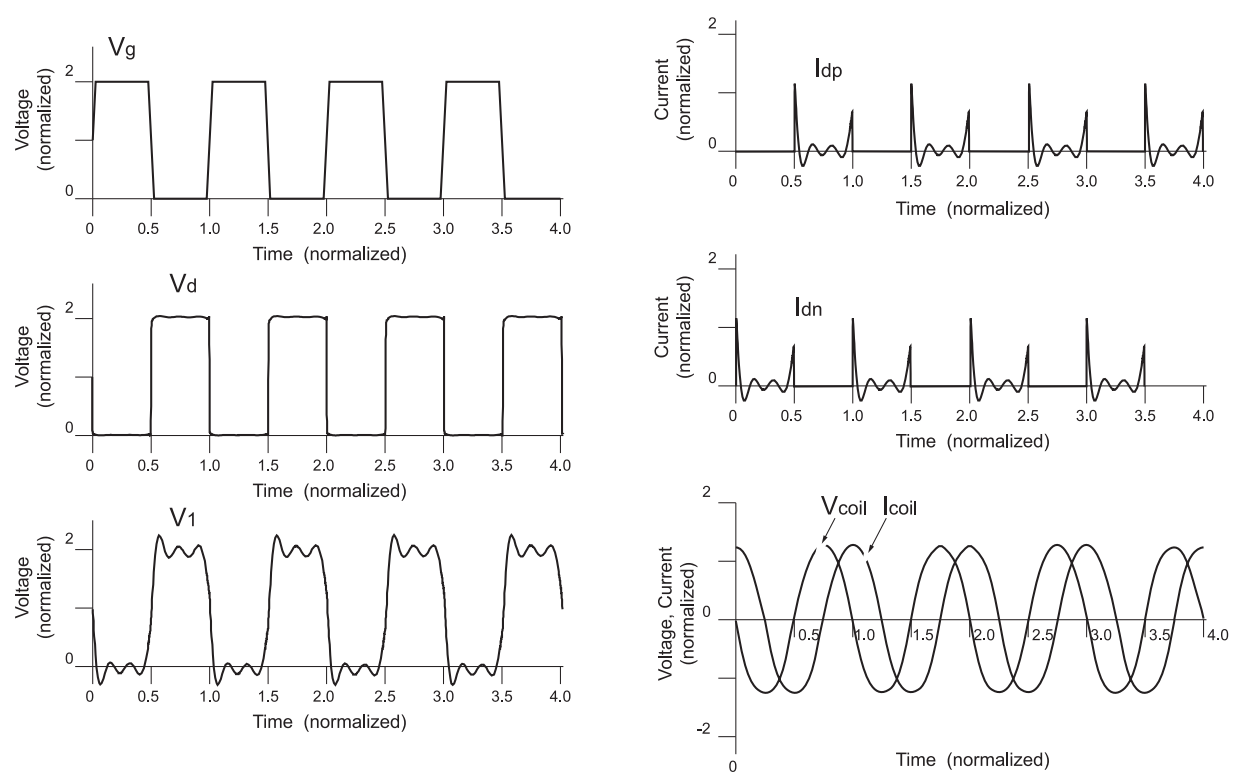

(b) Voltage and current waveform

Fig. 1. Transmitter design for inductive data link with class-F switching operation.

resonance frequency, the series LC resonators create a high impedance to the amplifier at the respective odd harmonic frequencies. Because of the weak magnetic coupling, the power loss due to the coupling to the receiver coil is negligible.

\subsection{Circuit operation}

Fig. 1 (b) illustrates voltage and current waveforms of the proposed transmitter. When a digital carrier signal with a $50 \%$ duty cycle square waveform $\left(V_{g}\right)$ is applied to the driver input. An inverted square waveform voltage appears at the CMOS inverter output $\left(V_{d}\right)$. A square wave of carrier frequency $\omega_{c}$ contains the fundamental frequency and all odd harmonics of the 
fundamental, and is expressed as

$$
V_{d}=\frac{2 V_{D D}}{\pi} \cdot \sum_{k=1}^{\infty}\left[\frac{\sin \left((2 k-1) \omega_{c} t\right)}{2 k-1}\right]+\frac{V_{D D}}{2} .
$$

By removing harmonics from the square drain-voltage waveform, this circuit generates an accurate sine waveform voltage $V_{\text {coil }}$ and current $I_{\text {coil }}$ at the output LC tank as

$$
\begin{gathered}
V_{\text {coil }}=\frac{2 V_{D D}}{\pi} \sin \omega_{c} t \\
I_{\text {coil }}=\frac{V_{\text {coil }}}{j \omega_{c} L_{1}}=-\frac{2 V_{D D}}{\pi \cdot \omega_{c} \cdot L_{1}} \cos \omega_{c} t .
\end{gathered}
$$

The quality factor of the class-F transmitter is given by

$$
Q=\frac{R}{\omega_{c} \cdot L_{1}}
$$

Therefore, $I_{\text {coil }}$ is expressed as

$$
I_{\text {coil }}=-\frac{2 V_{D D} \cdot Q}{\pi \cdot R} \cos \omega_{c} t
$$

The quality factor is chosen so as to meet the required bandwidth of the baseband signal. Therefore, the driving current for the transmitter coil is regulated by the resistance $\mathrm{R}$.

The node voltage $V_{1}$ is expressed by summation of the first through $N$ th odd harmonics as

$$
V_{1}=\frac{2 V_{D D}}{\pi} \cdot \sum_{m=1}^{N}\left[\frac{\sin \left((2 m-1) \omega_{c} t\right)}{(2 m-1)}\right]+\frac{V_{D D}}{2} .
$$

\section{Estimation of the transmitter power consumption}

Fig. 2 illustrates steady-state current waveforms $I_{R}$ flowing through the resistor $\mathrm{R}$ for various numbers of odd harmonics tuning stages. The waveforms are normalized to the maximum dc current $I_{\max }$ for the case without resonators given by

$$
I_{\max }=\frac{V_{D D}}{2 R} .
$$

Fig. 2 (a) shows the waveform without resonators. Time scale is normalized to the period of the carrier frequency, $T_{c}$. Fig. 2 (b) shows the waveform with an output LC tank tuned to only fundamental frequency. Waveforms with a number of parallel resonators connected in series are shown in Fig. 2 (c)-(f). Odd harmonic resonators reduce the power consumption due to the resistance $\mathrm{R}$ for the regulation of the transmitter coil current.

In the case that carrier signal only is applied, the power consumption of the transmitter $P_{\text {carrier }}$ is given by

$$
P_{\text {carrier }}=\frac{1}{T_{c}} \int_{-\frac{T_{c}}{2}}^{\frac{T_{c}}{2}} \frac{\left(V_{d}-V_{1}\right)^{2}}{R} d t .
$$

With Eqs. (1) and (6), it is calculated as

$$
P_{\text {carrier }}=\frac{8}{\pi^{2}} \cdot \frac{V_{D D}^{2}}{4 R} \cdot \sum_{k=N+1}^{\infty} \frac{1}{(2 k-1)^{2}} .
$$




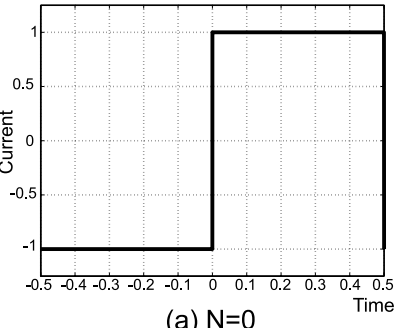

(a) $\mathrm{N}=0$

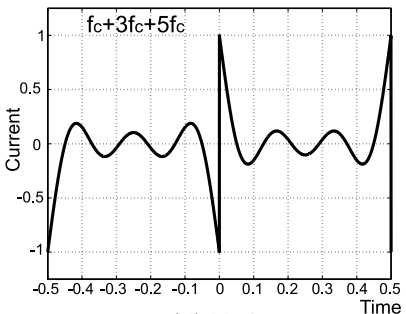

(d) $\mathrm{N}=3$

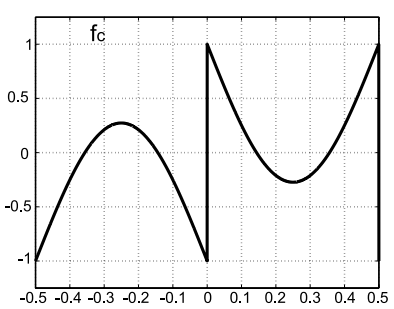

(b) $\mathrm{N}=1$

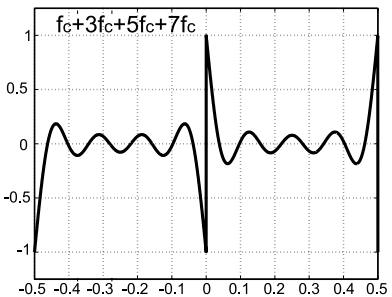

(e) $\mathrm{N}=4$

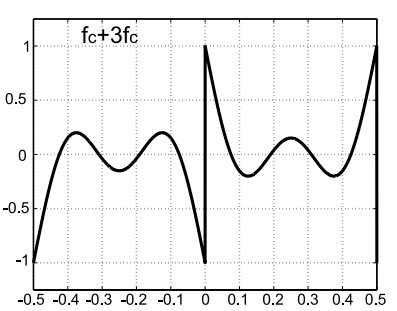

(c) $\mathrm{N}=2$

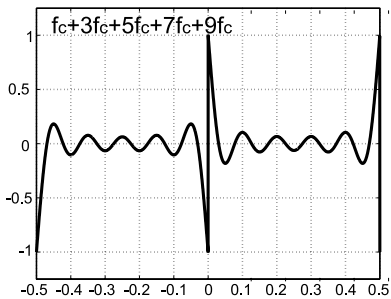

(f) $\mathrm{N}=5$

Fig. 2. Current waveforms of the transmitter for $\mathrm{N}=0 \sim 5$.

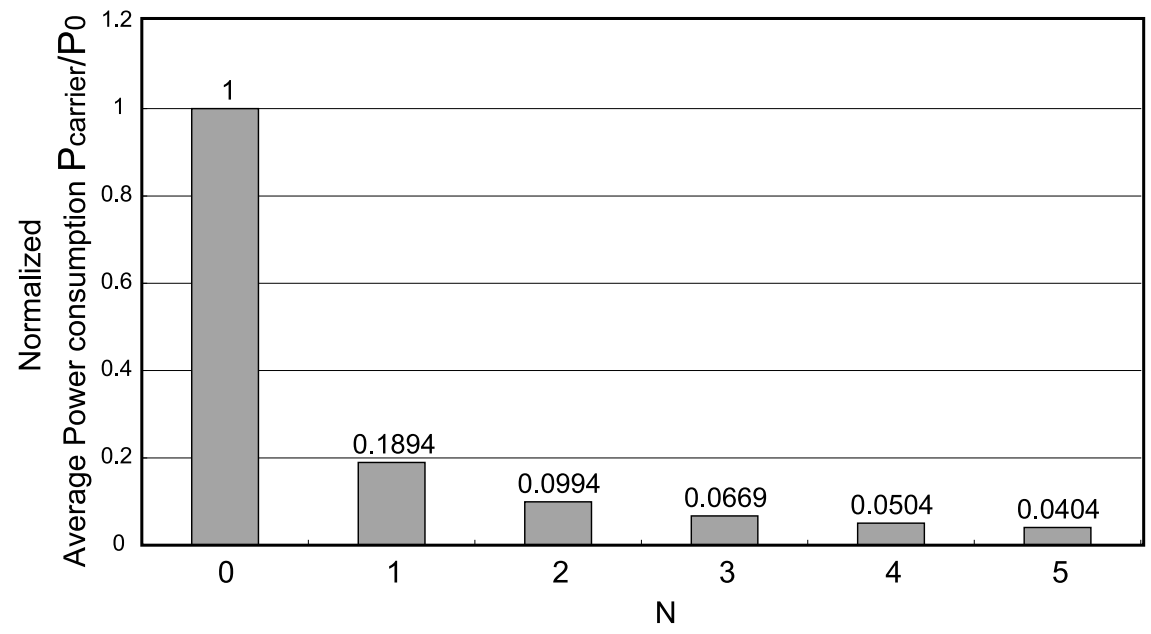

Fig. 3. Carrier power loss of the transmitter with the $N$ th order odd harmonic resonators.

The power dissipation without the resonators is given by

$$
P_{0}=\frac{V_{D D}^{2}}{4 R} \text {. }
$$

Fig. 3 shows the carrier power loss for the class-F transmitter with different odd harmonic resonators. The power consumption is normalized by $P_{0}$. As the number of odd harmonic resonators increases, the transmitter power efficiency can be significantly increased.

The power is also dissipated by the transient current to charge or discharge each capacitor of LC resonators for symbol transition. In the case of BPSK modulation considered here, the energy is consumed when the symbol goes from positive to negative or vice-versa. The dynamic energy consumption $E_{\text {symbol }}$ to charge and discharge the capacitors due to symbol transition is approximately given by

$$
E_{\text {symbol }}=\sum_{m=1}^{N}\left[\frac{1}{2} \cdot \frac{C_{1}}{2 m-1} \cdot\left(2 \times \frac{2 \cdot V_{D D}}{(2 m-1) \cdot \pi}\right)^{2}\right]
$$




$$
=\frac{8 \cdot C_{1} \cdot V_{D D}^{2}}{\pi^{2}} \sum_{m=1}^{N} \frac{1}{(2 m-1)^{3}} .
$$

The power consumption due to symbol transition $P_{\text {symbol }}$ is given by

$$
P_{\text {symbol }}=E_{\text {symbol }} \cdot f_{s} \cdot \alpha_{s}
$$

where $f_{s}$ is the symbol rate, and $\alpha_{s}$ is the symbol transition probabilities. The total power consumption is given by

$$
P_{\text {total }}=P_{\text {carrier }}+P_{\text {symbol }} .
$$

Table I shows the simulation results for the estimated power consumption of the proposed transmitter with different number of odd harmonic resonators. This simulation assumes a BPSK-modulated carrier signal with the carrier frequency $f_{c}$ of $20 \mathrm{MHz}$ and the symbol rate of $2.5 \mathrm{MSample} / \mathrm{s}$. The parameters shown in Table I correspond to the communication distance of $48 \mathrm{~cm}$ [6]. The lowest energy consumption can be achieved in ever-reported inductive data links for implantable systems.

Table I. Estimation of the transmitter power consumption.

$$
V_{D D}=2 \mathrm{~V}, \mathrm{Q}=8, \mathrm{R}=1 \mathrm{k} \Omega, L_{1}=1 \mu H, C_{1}=63 p F
$$

carrier frequency $f_{c}=20 \mathrm{M}[\mathrm{Hz}], f_{s}=2.5 \mathrm{M}[$ sample $/ \mathrm{s}], \alpha_{s}=0.5$

\begin{tabular}{|c|c|c|c|c|c|}
\hline $\mathrm{N}$ & $\begin{array}{c}\text { Odd } \\
\text { Harmonics }\end{array}$ & $\begin{array}{c}P_{\text {carrier }}[\mu W] \\
{[\mu W}\end{array}$ & $\begin{array}{c}P_{\text {symbol }}[\mu W] \\
{[\mu W]}\end{array}$ & $\begin{array}{c}P_{\text {total }} \\
{[\mu W \text { Energy Cost }} \\
{[\mathrm{nJ} / \text { bit }]}\end{array}$ \\
\hline 1 & 1 & 189 & 255 & 444 & 0.178 \\
\hline 2 & 1,3 & 99 & 265 & 364 & 0.146 \\
\hline 3 & $1,3,5$ & 67 & 267 & 334 & 0.134 \\
\hline 4 & $1,3,5,7$ & 50 & 268 & 318 & 0.127 \\
\hline 5 & $1,3,5,7,9$ & 40 & 268 & 308 & 0.123 \\
\hline
\end{tabular}

\section{Conclusion}

A new design method of an extremely low-power transmitter which introduces the concept of class-F amplifiers into an inductive data link has been presented. As the number of odd harmonic resonators increases, the transmitter power efficiency can be significantly increased by class-F switching operation. This extremely low-power consumption is useful for short range communications in implantable devices. 